\title{
New Polymer Design by DLS Analysis of Development Defect Detection
}

\author{
Yuki Kushida $^{\text {a) }}$, Yutaka Makita ${ }^{\text {a) }}$, Takanori Kawakami ${ }^{\text {b) }}$, Kenji Hoshiko ${ }^{\text {b) }}$, Hiroki Nakagawa ${ }^{\text {b) }}$, \\ Yukio Nishimura $^{\text {b) }}$ and Yoshikazu Yamaguchi ${ }^{\text {b) }}$ \\ a) Material Characterization \& Analysis Laboratory, JSR Corp, 100 \\ Kawajiri-cho, Yokkaichi, Mie, 510-8552 Japan \\ b)Semiconductor Materials Laboratory, Semiconductor Materials Laboratories, JSR Corp, 100 \\ Kawajiri-cho, Yokkaichi, Mie, 510-8552 Japan
}

\begin{abstract}
The behavior of developed ArF deprotected polymer to TMAH developer was demonstrated in this study to understand the effect of deprotected polymer's solubility and dispersion on development defect generation. The aggregation of deprotected polymer was observed with the dynamic light scattering (DLS) measurements. The observed aggregates were classified cluster- 1 and cluster- 2 based on the size, of which hydrodynamic radii $\left(R_{H}\right)$ were c.a. $100 \mathrm{~nm}$ and more than $500 \mathrm{~nm}$, respectively. In addition, the size and existing ratio of the aggregates were amplified by being exposed PAG and diluted in the developer. Furthermore, structure of protecting group and monomer composition showed large impact on the size of the cluster. It was found that DLS analysis could explain a main root cause of development defect generation, which possibly is the cluster formation. According to this study, ArF polymer design was suggested with development defect free as new concept for future ArF resist technology.
\end{abstract}

Keywords: ArF resist, Development defect, Dynamic light scattering (DLS), Cluster

\section{Introduction}

Sub $50 \mathrm{~nm}$ lithographic technology in the semiconductor industry has continued to progress year by year. Correspondingly, photoresist, a key material used in the lithographic technology, has been developed intensively according to the requirements of semiconductor manufacturers. The photoresist used for ArF lithography is mainly based on acrylic polymer because of its high transmittance at the $193 \mathrm{~nm}$ of wavelength, long-term stability and excellent lithographic performance. In addition to the improvement of lithographic performance, the countermeasure to various defects [1-4], such as so called "development defect" $[5,6]$, is important for achieving a reliable lithographic process. These development defect matters seem to be attached to the surface of pattering profiles during the developing process. Therefore, it is assumed that the development defect must be caused by low solubility of deprotected polymer dispersed in developer and this low solubility may be one of main factors to form development defect. However, we are aware of few studies on the development defect caused by low solubility of polymer in the developer.

In order to elucidate a mechanism of development defect, it is necessary to know the solution properties of the deprotected polymer dispersed in the developer. Dynamic light scattering (DLS) is a powerful tool for studying the dynamics of polymer solutions and is widely used in various researches of solution properties. Especially when applied to the defect problem, it was expected to make clear the little-known dispersion state of the deprotected polymer in developer. In a preliminary trial of DLS analysis, large aggregates were found in used developer collected by a manual developing procedure. It was considered that these aggregates would lead to 
the generation of development defect.

In this paper, we address the defect problem by utilizing a new analytical method using DLS and propose a design concept of defect-free photoresist based on the study of deprotected polymer in the developer. The polymer composition dissolved into developer is generally non-homogeneous owing to the polymer dissolution mechanism. In order to understand the effect of the polymer composition, model polymers with a fixed deprotected content were prepared. We will also discuss the effect of rinse process on the deprotected polymer's solubility.

\section{Method}

\subsection{Materials}

All model polymers (polymer-A to E in Table 1) were synthesized by free radical polymerization with azobisisobutylonitrile (AIBN). The MA unit was an idealized deprotected group after exposure and PEB. Monomer structures and polymer compositions are summarized in Figure 1 and Table 1. Three types of photo acid generators (PAGs), PAG-A, PAG-B, and PAG-C were used. $2.38 \mathrm{wt} \%$ of tetramethylammonium hydroxide (TMAH) aqueous solution was used as developer, and pure water, filtrated through a Millipore filter, was used as a dilution solvent.

\subsection{Sample preparations}

The coating and baking were conducted on an in-line track, Clean Track ACT8 (Tokyo Electron Inc.). Coated silicon wafers were exposed on a Nikon S306C ArF scanner through quartz with conventional illumination. Sample solutions were prepared by dissolving polymer and PAG in a solvent which was a mixture of cyclohexanone (CHX) and propylene glycol methyl ether acetate (PGMEA) with a ratio of 7:3. These samples were coated on 8-inch silicon wafers, and pre-baked at $100^{\circ} \mathrm{C}$ for 60 seconds. The thickness of the resist films was approximately $500 \mathrm{~nm}$. In order to ensure high concentration for later analysis, three resist films were prepared for each sample, and were developed with $10 \mathrm{cc}$ of developer by a manual method.

\subsection{DLS analysis}

DLS measurements $[7,8]$ were carried out to evaluate the hydrodynamic radius $\left(\mathrm{R}_{\mathrm{H}}\right)$ for all the samples in developer at $23^{\circ} \mathrm{C}$, using an ALV/DLS/SLS-5000 light scattering system equipped by an ALV-5000 multiple tau digital

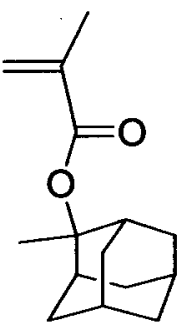

monomer-A

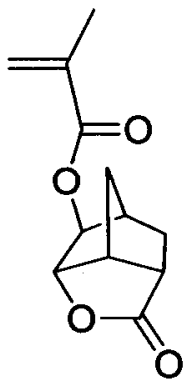

monomer-C

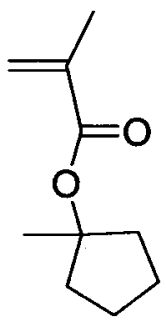

monomer-B

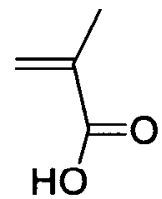

MA

Fig. 1 Monomer structures

Table 1 Composition of model polymers

\begin{tabular}{ccccc} 
& \multicolumn{4}{c}{ monomer ratio(\%) } \\
\cline { 2 - 5 } polymer & monomerA & monomerB & monomerC & MA \\
\hline polymer-A & 20 & - & 50 & 30 \\
polymer-B & 30 & - & 50 & 20 \\
polymer-C & 40 & - & 50 & 10 \\
polymer-D & 50 & - & 50 & 0 \\
polymer-E & - & 20 & 50 & 30
\end{tabular}

correlator. Vertically polarized light with the wavelength of $632.8 \mathrm{~nm}$ emitted from a $\mathrm{He}-\mathrm{Ne}$ laser was used as the incident light, and the scattered light was measured with no analyzer. The normalized auto-correlation function $g^{(2)}(t)$ of the scattered light intensity at sampling time $t$ was measured at a fixed scattering angle of $60^{\circ}$. The sample solution was optically purified by filtration through a Teflon membrane with a $1.0 \mu \mathrm{m}$ pore size. The polymer mass concentration $c$ (in $\mathrm{g} / \mathrm{cm}^{3}$ ) was estimated from the refractive index of the solution using calibration curve of model polymers. The conc-entrations of sample solutions ranged from $1 \times 10^{-5}$ to $5 \times 10^{-4}$ and were in the dilute solution region.

The translational diffusion coefficient $D$ was determined by analyzing auto-correlation function data and converted to the hydrodynamic radius $R_{H}$ using the Stokes-Einstein equation $R_{H}=k_{B} T / 6 \pi \eta_{0} D$. The viscosity coefficient $\eta_{0}$ of solvent at $23.0^{\circ} \mathrm{C}$ was determined with a rotational viscometer (Toki Sangyo Co. RE-80L) to be 0.89 $\mathrm{cP}$. The sample solutions used in this study showed 
large polydispersity of $\mathrm{R}_{\mathrm{H}}$. Therefore, the distribution of $R_{H}$ was analyzed with the CONTIN method [9].

\section{Results and discussion}

\subsection{Unexposed state samples}

Unexposed state samples for a blank test were prepared as follows. $1 \mathrm{wt} \%$ developer solution of polymer-A with or without PAG were prepared. Polymer-A has a high content of MA units - deprotected group - and easily dissolved in developer. Furthermore, it was diluted by pure water to simulate a rinse process after developing. It is desirable to dilute the solution to a near-neutral condition with a large quantity of pure water. However, a scattering intensity enough for DLS measurement is not attained when the dilution is too high. We selected a 50 times dilution by pure water as the highest dilution to maintain the required scattering intensity.

Fig. 2a) shows DLS analysis of unexposed state samples. Two peaks, a main peak around $3 \mathrm{~nm}$ and a small peak around $100 \mathrm{~nm}$, were observed in both the polymer-A and polymer-A/PAGs samples. The addition of PAG did not affect the distribution of $\mathrm{R}_{\mathrm{H}}$. Similarly two peaks were also observed in the diluted samples, but the second peak, around $100 \mathrm{~nm}$, increased significantly as shown in Fig. 2b). It was thought that the first peak, around $3 \mathrm{~nm}$, was ascribed to single polymer chains, and the second peak around $100 \mathrm{~nm}$ to polymer aggregates. The second peak was called cluster- 1 . The mechanism of cluster formation is not clear at this moment, but it is estimated that polymer chains with high molecular weight or low content MA units would easily aggregate owing to low solubility in developer. The reason why cluster- 1 increased in diluted samples is that the decrease of alkali concentration made polymer's solubility lower.

The weight fraction of cluster-1 can be evaluated by relative scattering intensity. Scattering intensity in DLS measurement is calculated as z-averaged quantity, which means scattering intensity is proportional to the square of molecular weight $(M)$. The weight fraction $(w)$ of cluster- 1 is roughly estimated from the relative scattering intensity of cluster- 1 to that of the main peak $(\alpha)$ by the following equation, $w=\alpha M_{1} / M_{2}$. $M_{1}$ and $M_{2}$ are the weight-averaged molecular weight of single polymer chains and cluster- 1 respectively. Cluster- 1 is considered to be a uniform sphere consisting of many polymer chains
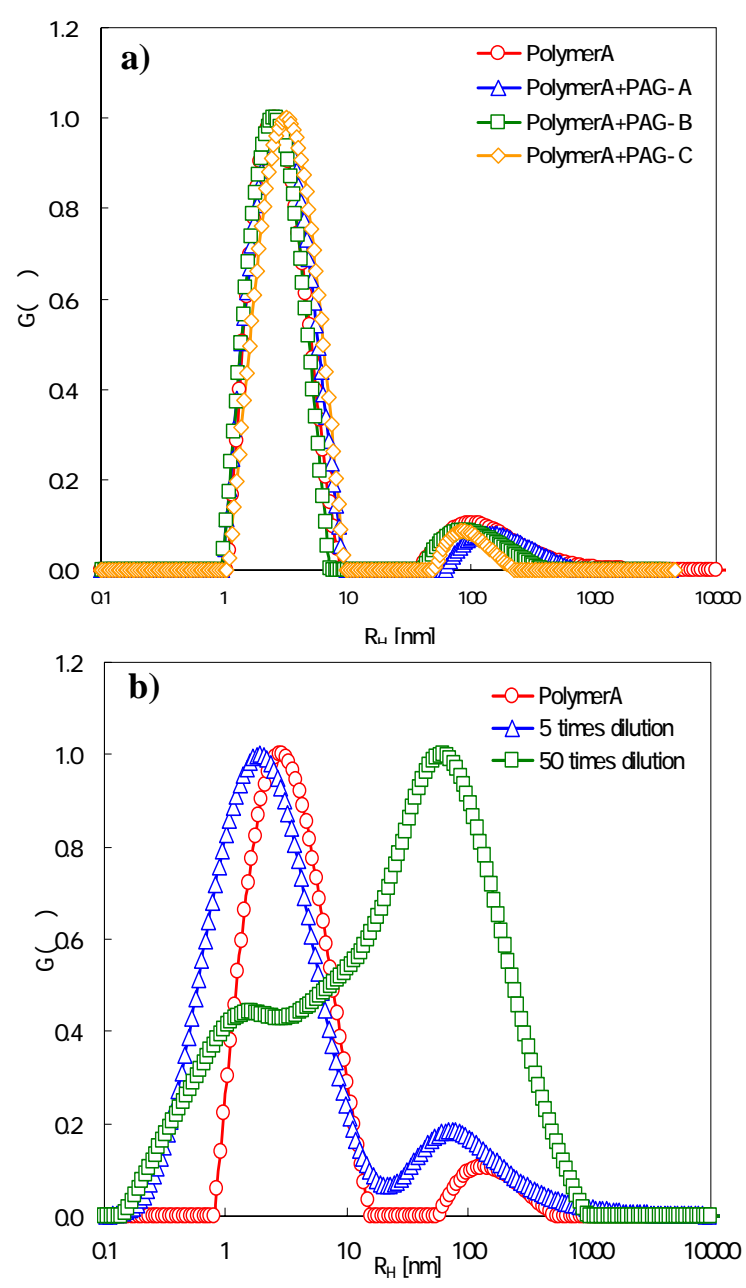

Fig. 2 DLS analysis of unexposed samples a)Polymer-A and polymer-A w/ PAG samples b)Diluted samples of Polymer-A ; 5 times dilution and 50 times dilution by pure water

and the relation between $M$ and $\mathrm{R}_{\mathrm{H}}$ is expected to be $M \propto R_{H}^{3}$. Therefore we obtain the relation, $w=a\left(R_{H 1} / R_{H 2}\right)^{3}$. The estimated weight fraction of cluster-1, using this relation, was about $3 \mathrm{ppm}$, here we neglected the effect of internal interference in the large cluster, so the true value somewhat bigger. It was concluded that most of the polymer chains uniformly dissolved in the developer and a small number of polymer chains with low solubility formed the large cluster.

\subsection{Exposed state samples}

The influence of exposure on the solubility of the deprotected polymer was investigated, but the baking after exposure was omitted to keep the content of deprotected groups unchanged. The exposed state samples were prepared from polymer-A using the same method as described in 

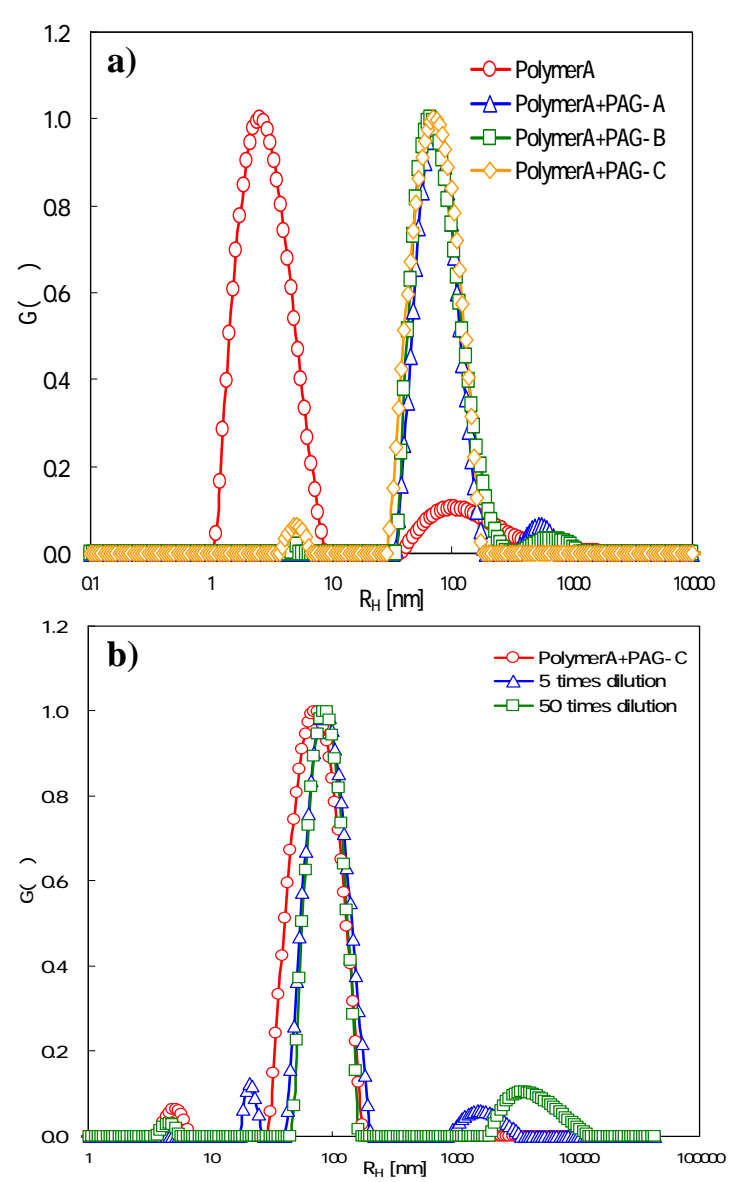

Fig. 3 DLS analysis of exposed samples a)Polymer-A and polymer-A w/ PAG samples b)Diluted samples of Polymer-A w/ PAG-C; 5 times dilution and 50 times dilution by pure water

\section{Section 3.1.}

Fig. 3 a) shows the DLS analysis of the exposed state samples. As for the sample without PAG, the distribution of $R_{H}$ was nearly same as that of the unexposed state sample. However, in the case of samples with PAGs, the cluster-1 peaks increased greatly and the polymer peaks became negligible. Furthermore, a third peak, just above $500 \mathrm{~nm}$, was observed in samples with PAG-A and PAG-B. This new peak was called cluster-2. In diluted samples with PAGs, cluster-2 increased in size and amount. Even in sample with PAG-C, in which a cluster-2 peak was not previously observed, a cluster- 2 peak was generated in the dilution process as shown in Fig. $3 \mathrm{~b}$ ). It is considered that the significant increase of cluster- 1 in samples with PAG was caused by the photo-decomposed products of PAG, including generated acids. The amount of cluster- 1 was estimated to be more than $1000 \mathrm{ppm}$. It is noted that cluster-1 increased more than a thousand fold compared to that in unexposed samples.
Table 2 Summary of mixed ratio of model polymers

\begin{tabular}{ccc} 
Mixtured Polymer & Mixtured ratio & A:C:MA \\
\hline polymer-A and B & $75: 25$ & $22.4: 50: 27.6$ \\
polymer-A and C & $87.5: 12.5$ & $22.4: 50: 27.6$ \\
polymer-A and D & $92: 8$ & $22.4: 50: 27.6$
\end{tabular}

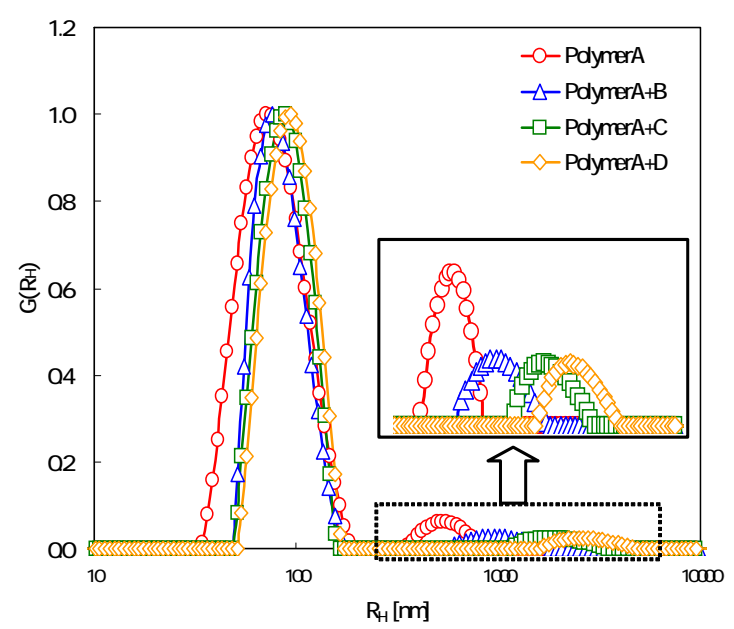

Fig. 4 DLS analysis of blended polymer samples. The mixture ratio was summarized in Table 2.

In conclusion, the exposed samples with PAGs caused a large amount of cluster- 1 and another, bigger cluster, cluster-2. The formation of this cluster in the developing and rinse process was regarded as a main factor in creating development defects.

\subsection{Mixture of polymers}

The mixture samples were prepared by blending polymer-A with another polymer with lower MA unit content (polymer-B, polymer-C, polymer-D) in order to investigate the effect of the deprotected group distribution. Although these polymers could not dissolve in developer because of insufficient content of deprotected group, mixed samples with polymer-A could dissolve into developer due to the high solubility of polymer-A. This phenomenon indicates that some portion of low deprotected polymer is possibly extracted with high solubility polymer into the developer, and possibly accelerates the cluster formation.

The mixture ratio was set in such a way as the MA unit content was equal to $27 \mathrm{~mol} \%$ in each sample. The mixture ratio is listed in Table 2 and the results of the DLS analysis are shown in Figure 3. A peak of single polymer chains was hardly detected in any of the mixed samples, and the size of cluster-2 increased when lower deprotected polymer was blended with polymer-A. Therefore, 


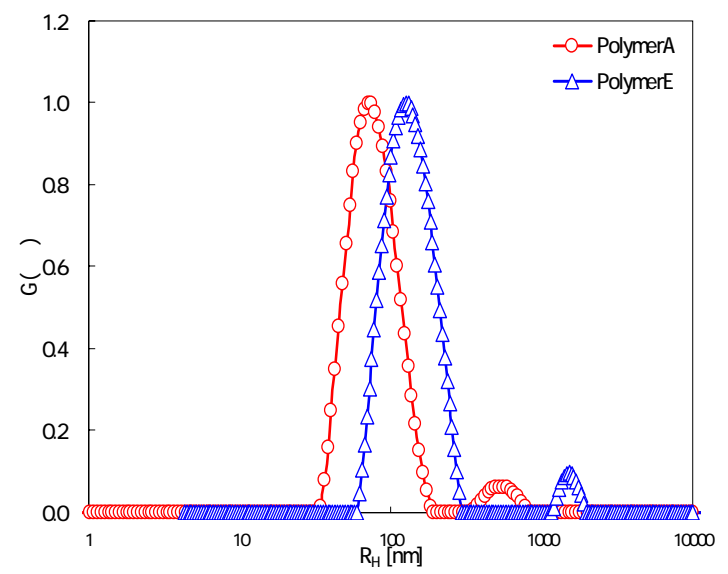

Fig. 5 Comparison of model polymers with different structures of protecting groups; polymer-A and polymer-E with PAG-A

it was supposed that lower deprotected polymers tended to become the nucleus for large cluster. It is considered that the most important thing to avoid unfavorable cluster formation is to understand a mechanism of the polymer-dissolution process.

\subsection{Influence of protecting group}

The $\mathrm{R}_{\mathrm{H}}$ distributions of deprotected polymers with different protecting groups in developer were compared in Fig. 5. The samples comprised polymer-A or polymer-E with PAG-A, and were exposed before developing as described in section 3.2. Two types of cluster, cluster- 1 and cluster-2, were observed in polymer-A and were also confirmed in polymer-E, however, both cluster peaks became larger than those in polymer-A. A defect test was also conducted on the same samples. The number of defects detected in the polymer-E sample was much higher than that in the polymer-A sample. Clearly, as the cluster size increased, the number of defects also increased.

The reason why polymer-E caused so many defects and large cluster in developer has not been clearly understood yet. In fact, the solubility of polymer-E in developer was higher than that of polymer-A due to the size of protecting group. Therefore, it was supposed that it important to precisely control the solubility of polymer into developer by the design of polymer composition, polymer sequence, and chemical structure.

\subsection{Proposed mechanism of development defect}

Through the DLS analysis of model deprotected polymers in developer, the following outputs were gained for understanding

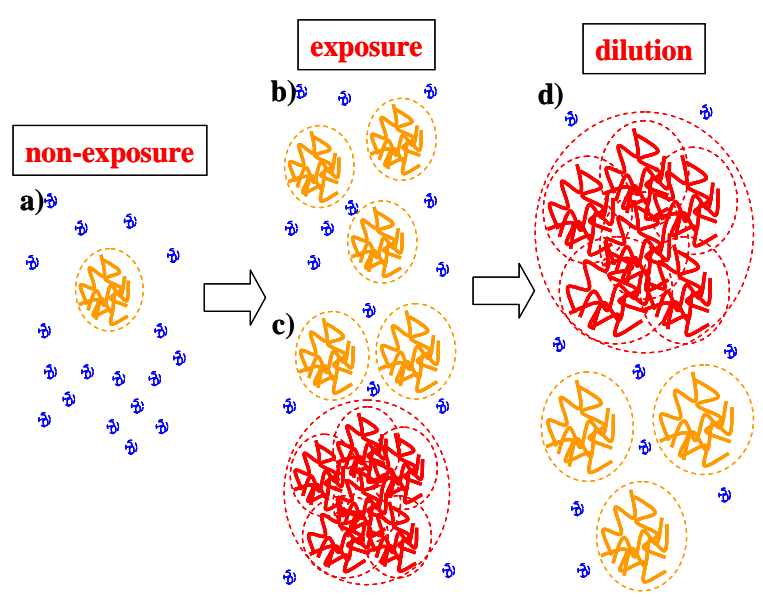

Fig. 6 Image of cluster formation in developing process

a) unexposed: small amount of cluster-1, b) exposed: formation of cluster-1, c) exposed: formation of cluster-1 and 2, d) dilution by rinse process: growth of cluster

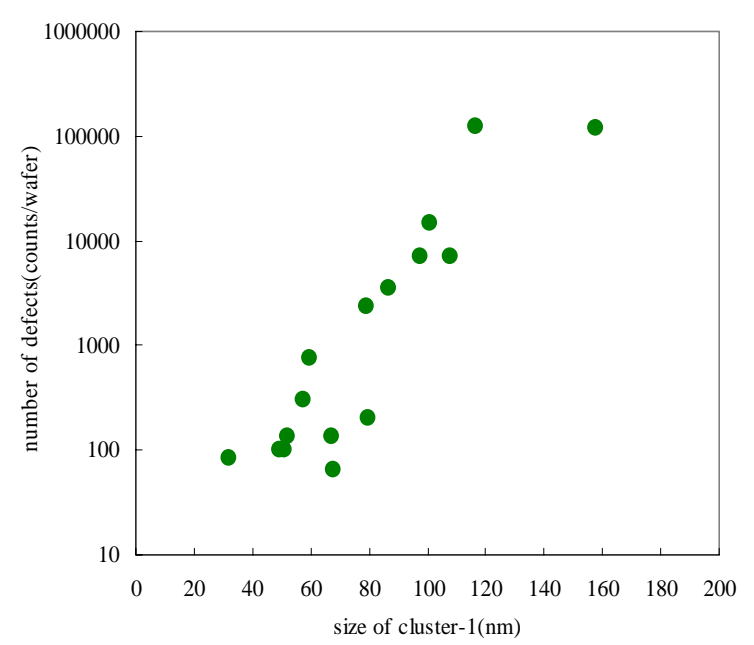

Fig. 7 Relations between the number of development defects and $R_{H}$ of cluster- 1

development defect generation.

1) The deprotected polymers can easily dissolve into developer as single polymer chains. Aggregation was observed, but the amount was small.

2) The aggregation of deprotected polymers, which is considered to be the main cause of development defect, is accelerated by the photo-decomposed PAG.

3) The size and amount of cluster are influenced by the structure of protecting group and the polymer composition in the developer.

4) The cluster formation is also significantly accelerated by diluting developer which simulates a rinse process.

The image of cluster formation mechanism is 
illustrated in Fig. 6. The formed cluster is supposed to have lower solubility in developer and relatively high affinity to the resist film and substrate. The relation between the number of development defects and the size of cluster- 1 in various photoresist samples is shown in Fig. 7, in which a clear correlation is confirmed.

According to these investigations on deprotected polymers in developer, it was concluded that cluster formation in developer was a main cause of development defect. Furthermore, a new photopolymer was designed with less development defect by controlling the solubility and dissolution of the polymers in developer.

\section{Conclusions}

In this paper, DLS analysis of deprotected polymer in developer has been done in order to elucidate the main factors of development defect in photolithography. It was found that cluster of deprotected polymers was an origin of development defect. The cluster formation was accelerated by photo-decomposed PAG and dilution in developer, and it was concluded that a main cause of development defect was the attachment of cluster resist film and substrate. In addition, we determined that the size and amount of cluster were influenced by the structure of protecting group and polymer composition in the developer. Finally, an imagable mechanism of development defect generation and a new polymer design with less development defects were proposed based on these investigations.

\section{References}

1.K. M. Kim, Y. Yoon, J. H. Kim, Y. H. Kim, S. M. Chon, J. photopolym. Sci. Technol., 17(2004), 545

2. H. Endo, A. Kawai, J. photopolym. Sci. Technol, 17(2004), 105

3. H. Endo, A. Kawai, J. photopolym. Sci. Technol., 17(2004), 713

4. S. Kanna, H. Inabe, K. Yamamoto, T. Fukuhara, S. Tarutani, H. Kanda, K. Wada, K. Kodama, K.

Shitabatake. J. photopolym. Sci. Technol., 19(2006), 593

5. S. Skordas, R. L. Burns, D. L. Goldfarb, S. D. Burns, M. Angelopoulos, C. J. Brodsky, M. C. Lawson, C. J. Pillette, J. J. Bright, R. L. Isaacson, M. E. Lagus, V. Vishnu, Proc. SPIE, 5376 (2004), 471

6. Y. Shen, E. Y. Lam, Proc. SPIE, 6070 (2006)

7. B. J. Berne, R. Pecora Dynamic Light Scattering, Dover Publication, Inc (2000)

8. H. C. van de Hulst Light Scattering by Small Particles, Dover Publication, Inc (1981)

9. Provencher, S. W. Biophys. J. 1976, 16, 29. 